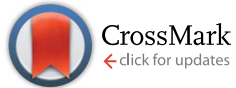

Cite this: RSC Adv., 2017, 7, 15352

Received 11th January 2017

Accepted 23rd February 2017

DOI: $10.1039 / \mathrm{c} 7 \mathrm{ra00447h}$

rsc.li/rsc-advances

\section{Magnetoliposomes as carriers for promising antitumor thieno[3,2-b]pyridin-7-arylamines: photophysical and biological studies $\uparrow$}

\author{
Ana Rita O. Rodrigues, ${ }^{a}$ B. G. Almeida, ${ }^{a}$ Juliana M. Rodrigues, ${ }^{b}$ \\ Maria João R. P. Queiroz, ${ }^{b}$ R. C. Calhelha, ${ }^{c}$ Isabel C. F. R. Ferreira, ${ }^{c}$ A. Pires, ${ }^{d}$ \\ A. M. Pereira, ${ }^{d}$ J. P. Araújo, ${ }^{d}$ Paulo J. G. Coutinho ${ }^{a}$ and Elisabete M. S. Castanheira*a
}

Magnetoliposomes containing superparamagnetic manganese ferrite nanoparticles were tested as nanocarriers for two new promising antitumor drugs, a N-(3-methoxyphenyl)thieno[3,2-b]pyridin-7-amine (1) and a N-(2methoxy-phenyl)thieno[3,2-b]pyridin-7-amine (2). The fluorescence emission of both compounds was studied in different polar and non-polar media, evidencing a strong intramolecular charge transfer character of the excited state of both compounds. These in vitro potent antitumor thienopyridine derivatives were successfully incorporated in both aqueous and solid magnetoliposomes, with encapsulation efficiencies higher than $75 \%$. The magnetic properties of magnetoliposomes containing manganese ferrite nanoparticles were measured for the first time, proving a superparamagnetic behaviour. Growth inhibition assays on several human tumor cell lines showed very low $\mathrm{Gl}_{50}$ values for drug-loaded aqueous magnetoliposomes, comparing in most cell lines with the ones previously obtained using the neat compounds. These results are important for future drug delivery applications using magnetoliposomes in oncology, through a dual therapeutic approach (simultaneous chemotherapy and magnetic hyperthermia).

\section{Introduction}

The high potential of magnetic nanoparticles for biomedical applications has been widely recognized due to their unique size and physicochemical properties. Nanoparticles with superparamagnetic behavior are preferred in biomedicine, as they exhibit a strong magnetization only when an external magnetic field is applied., ${ }^{\mathbf{1} 2}$

Liposomes entrapping magnetic nanoparticles (magnetoliposomes) are of large importance in drug delivery, as they can be guided and localized to the therapeutic site of interest by external magnetic field gradients and used in cancer treatment by hyperthermia. ${ }^{3,4}$ Magnetoliposomes have been proposed as T2 contrast agents (negative contrast enhancement) in MRI, ${ }^{5}$ while in therapy they have been used as a chemotherapy

${ }^{a}$ Centro de Física (CFUM), Universidade do Minho, Campus de Gualtar, 4710-057 Braga, Portugal. E-mail: ecoutinho@fisica.uminho.pt; Fax: +351 253604061; Tel: +351253604321

${ }^{b}$ Centro de Química (CQUM), Universidade do Minho, Campus de Gualtar, 4710-057 Braga, Portugal

${ }^{c}$ Centro de Investigação Montanha (CIMO), ESA, Polytechnic Institute of Bragança, Campus de Sta Apolónia, 5301-855 Bragança, Portugal

${ }^{d}$ IFIMUP/IN - Instituto de Nanociência e Nanotecnologia, R. Campo Alegre, 4169-007 Porto, Portugal

$\dagger$ Electronic supplementary information (ESI) available: Size distribution of DPPC SMLs obtained by DLS; SEM image of DPPC SMLs; inhibitory activity of SMLs in cell lines. See DOI: 10.1039/c7ra00447h alternative through magnetic-controlled drug delivery and thermotherapy. ${ }^{6-8}$ Considering the wide applications of magnetoliposomes, much attention has been paid to the synthesis of different kinds of magnetic nanoparticles ${ }^{9-11}$ and liposomes. ${ }^{\mathbf{1 2 - 1 5}}$

Magnetoliposomes are specially promising as nanocarriers for potential antitumor drugs. Thienopyridine derivatives have been reported as possessing antiangiogenic, ${ }^{16-21}$ antitumor, ${ }^{21-29}$ or both activities. ${ }^{30}$ Among the potential antitumor di(hetero)arylamines in the thieno[3,2- $b]$ pyridine series, prepared earlier by some of us, the ones with an $o$-methoxy or $m$-methoxy groups relative to the $\mathrm{NH}$ (compounds 1 and 2, Fig. 1) presented the lowest growth inhibitory concentration $\left(\mathrm{GI}_{50}\right)$ values in several human tumor cell lines, between 0.09 and $0.31 \mu \mathrm{M}$ for compound 1 and between 1.40 and $5.91 \mu \mathrm{M}$ for compound $2 .^{29}$ Compound 1 revealed to be more active<smiles>COc1cccc(Nc2ccnc3ccsc23)c1</smiles>

1<smiles>COc1ccccc1Nc1ccnc2ccsc12</smiles>

Fig. 1 Structure of compound 1, N-(3-methoxyphenyl)thieno[3,2-b] pyridin-7-amine, and compound 2, N-(2-methoxyphenyl)thieno[3,2b]pyridin-7-amine. 
than the well-known anticancer agent ellipticine against the cell lines MCF-7 (breast adenocarcinoma), NCI-H460 (non-small cell lung carcinoma), HepG2 (hepatocellular carcinoma), HCT15 (colon adenocarcinoma), and HeLa (cervical carcinoma), while compound 2 showed the lowest $\mathrm{GI}_{50}$ against HeLa cells. However, these compounds also presented low $\mathrm{GI}_{50}$ values in non-tumor PLP2 cells (1.94 $\mu \mathrm{M}$ for compound 1 and $6.56 \mu \mathrm{M}$ for compound 2). ${ }^{29}$

Considering future applications of these new compounds as antitumor drugs, they were incorporated in magnetoliposomes, either aqueous or solid, containing manganese ferrite nanoparticles, suitable for biomedical applications. ${ }^{31}$

Magnetoliposomes may constitute ideal nanocarrier systems for encapsulation and transport of these drugs, as they can be guided and localized in tumor cells by a magnetic field gradient, and act simultaneously as chemotherapy/hyperthermia agents in a synergistic approach, reducing drug dosage due to the dual therapeutic effect.

\section{Experimental}

All the solutions were prepared using spectroscopic grade solvents and ultrapure water (Milli-Q grade).

\section{Preparation of magnetoliposomes}

Manganese ferrite nanoparticles (NPs) were synthesized by the coprecipitation method, as previously described..$^{31}$ For magnetoliposomes preparation, the lipids egg yolk phosphatidylcholine (Egg-PC), dipalmitoylphosphatidylcholine (DPPC) and cholesterol (Ch), from Sigma-Aldrich, were used. For aqueous magnetoliposomes preparation, a $10 \mathrm{mM}$ lipid solution in ethanol was injected, under vigorous vortexing, to an aqueous solution of magnetic nanoparticles, above the melting transition temperature of the lipids (ethanolic injection method). ${ }^{32}$ After encapsulation, the ferrofluid was washed with water and purified by ultracentrifugation to remove all the non-encapsulated NPs.

Solid magnetoliposomes (SMLs) were prepared by a method previously developed. ${ }^{33}$ First, $10 \mu \mathrm{L}$ of the synthesized $\mathrm{MnFe}_{2} \mathrm{O}_{4}$ NPs were dispersed in $3 \mathrm{~mL}$ of water and centrifuged. Then, the deposited particles were dispersed in $10 \mu \mathrm{L}$ of water in an ultrasonicator, for one minute at $189 \mathrm{~W}$, and $3 \mathrm{~mL}$ of chloroform were added to the aqueous dispersion of NPs. After vigorous agitation, $165 \mu \mathrm{L}$ of a $20 \mathrm{mM}$ solution of dipalmitoylphosphatidylcholine (DPPC) were added under vortexing, to form the first lipid layer of the SMLs. The particles were washed twice by magnetic decantation with pure water, in order to remove the lipid that was not attached to the NPs. The second lipid layer was then formed by the injection of $165 \mu \mathrm{L}$ of DPPC (20 $\mathrm{mM}$ ), under vortexing, in a $3 \mathrm{~mL}$ aqueous dispersion of the particles with the first layer. The resulting SMLs were then washed and purified with ultrapure water by centrifugation.

Compounds 1 and $\mathbf{2}$ were incorporated into aqueous magnetoliposomes by the co-injection method, as already described..$^{34}$ In solid magnetoliposomes, the diarylamines $\mathbf{1}$ and $\mathbf{2}$ were incorporated by injection of an ethanolic solution together with the formation of the second lipid layer.

\section{Spectroscopic measurements}

General methods. Absorption spectra were recorded in a Shimadzu UV-3101PC UV-Vis-NIR spectrophotometer. Fluorescence measurements were performed using a Fluorolog 3 spectrofluorimeter, equipped with double monochromators in both excitation and emission and a temperature controlled cuvette holder. Fluorescence spectra were corrected for the instrumental response of the system.

The fluorescence quantum yields, $\Phi_{\mathrm{s}}$, were determined by the standard method (eqn (1)),,$^{35,36}$

$$
\Phi_{\mathrm{s}}=\left[\left(A_{\mathrm{r}} F_{\mathrm{s}} n_{\mathrm{s}}^{2}\right) /\left(A_{\mathrm{s}} F_{\mathrm{r}} n_{\mathrm{r}}^{2}\right)\right] \Phi_{\mathrm{r}}
$$

where $A$ is the absorbance at the excitation wavelength, $F$ the integrated emission area and $n$ is the refraction index of the solvents. Subscripts refer to the reference (r) or sample (s) compound. The absorbance value at excitation wavelength was always less than 0.1 , in order to avoid inner filter effects.

Fluorescence anisotropy measurements. The steady-state fluorescence anisotropy, $r$, is calculated by

$$
r=\frac{I_{\mathrm{VV}}-G I_{\mathrm{VH}}}{I_{\mathrm{VV}}+2 G I_{\mathrm{VH}}}
$$

where $I_{\mathrm{VV}}$ and $I_{\mathrm{VH}}$ are the intensities of the emission spectra obtained with vertical and horizontal polarization, respectively (for vertically polarized excitation light), and $G=I_{\mathrm{HV}} / I_{\mathrm{HH}}$ is the instrument correction factor, where $I_{\mathrm{HV}}$ and $I_{\mathrm{HH}}$ are the emission intensities obtained with vertical and horizontal polarization (for horizontally polarized excitation light).

FRET measurements. The formation of a DPPC bilayer around manganese ferrite nanoparticles was investigated by Förster Resonance Energy Transfer (FRET), using the labeled lipids NBD-C 6 -HPC (1-palmitoyl-2-\{6-[(7-nitro-2-1,3-benzoxadiazol-4-yl)amino]hexanoyl $\}$-sn-glycero-3-phosphocholine) and rhodamine B-DOPE ( $N$-(lissamine rhodamine B sulfonyl)1,2-dioleoyl-sn-3-phosphatidylethanolamine (ammonium salt)), both from Avanti Polar Lipids (Fig. 2).

FRET efficiency, $\Phi_{\text {RET }}$, defined as the proportion of donor molecules that have transferred their excess energy to acceptor molecules, can be obtained by taking the ratio of the donor integrated fluorescence intensities in the presence of acceptor $\left(F_{\mathrm{DA}}\right)$ and in the absence of acceptor $\left(F_{\mathrm{D}}\right)$ (eqn $\left.(3)\right),{ }^{37}$

$$
\Phi_{\mathrm{RET}}=1-\frac{F_{\mathrm{DA}}}{F_{\mathrm{D}}}
$$

The distance between donor and acceptor molecules can be determined through the FRET efficiency (eqn (4)),

$$
r_{\mathrm{AD}}=R_{0}\left[\frac{1-\Phi_{\mathrm{RET}}}{\Phi_{\mathrm{RET}}}\right]^{1 / 6}
$$

where $R_{0}$ is the Förster radius (critical distance), that can be obtained by the spectral overlap, $J(\lambda)$, between the donor emission and the acceptor absorption, according to eqn (5) and (6) (with $R_{0}$ in $\AA$, $\lambda$ in $\mathrm{nm}, \varepsilon_{\mathrm{A}}(\lambda)$ in $\mathrm{M}^{-1} \mathrm{~cm}^{-1}$ ), ${ }^{37}$

$$
R_{0}=0.2108\left[k^{2} \Phi_{\mathrm{D}}^{0} n^{-4} J(\lambda)\right]^{1 / 6}
$$



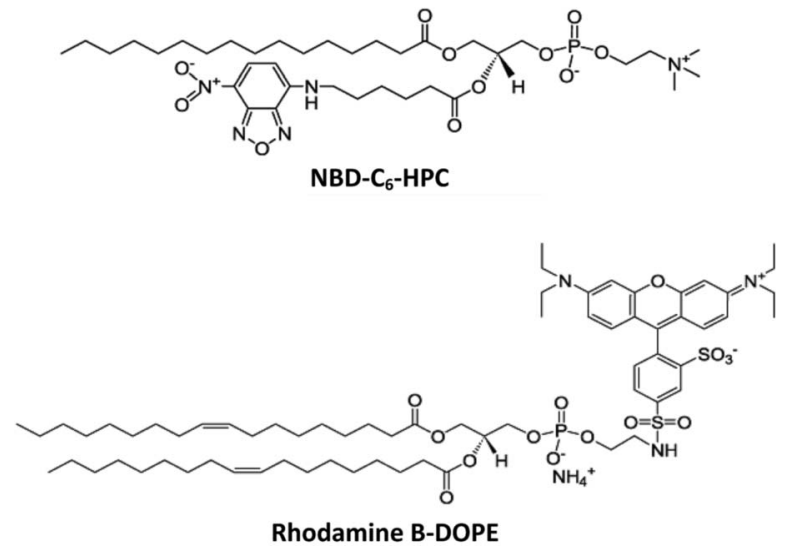

Fig. 2 Structure of the labeled lipids NBD- $\mathrm{C}_{6}-\mathrm{HPC}$ and rhodamine BDOPE.

$$
J(\lambda)=\int_{0}^{\infty} I_{\mathrm{D}}(\lambda) \varepsilon_{\mathrm{A}}(\lambda) \lambda^{4} \mathrm{~d} \lambda
$$

where $k^{2}=2 / 3$ is the orientational factor assuming random orientation of the dyes, $\Phi_{\mathrm{D}}^{0}$ is the fluorescence quantum yield of the donor in the absence of energy transfer, $n$ is the refraction index of the medium, $I_{\mathrm{D}}(\lambda)$ is the fluorescence spectrum of the donor normalized so that $\int_{0}^{\infty} I_{\mathrm{D}}(\lambda) \mathrm{d} \lambda=1$, and $\varepsilon_{\mathrm{A}}(\lambda)$ is the molar absorption coefficient of the acceptor.

For determination of fluorescence quantum yield of NBD-C ${ }_{6}-$ HPC (energy donor) in magnetoliposomes, this fluorescent labeled lipid incorporated in lipid membranes was used as reference, $\Phi_{\mathrm{r}}=0.32$ at $25{ }^{\circ} \mathrm{C}$, as reported by Invitrogen..$^{38}$

\section{Compound encapsulation efficiency}

The encapsulation efficiency, EE (\%), of antitumor compounds was determined through fluorescence emission measurements. After preparation, drug-loaded magnetoliposomes (MLs) were subjected to centrifugation at $11000 \mathrm{rpm}$ for 60 minutes. The supernatant was pipetted out and its fluorescence was measured, allowing to determine the compound concentration using a calibration curve previously obtained. Three independent measurements were performed for each system and standard deviations (SD) were calculated. The encapsulation efficiency of compounds $\mathbf{1}$ and $\mathbf{2}$ was determined using the following equation:

$$
\mathrm{EE}(\%)=
$$

(total amount - amount of non encapsulated compound) total amount $\times 100$

\section{Scanning electron microscopy (SEM)}

Scanning electron microscopy (SEM) images of solid magnetoliposomes were recorded using a Scanning Electron Microscope FEI - Nova 200 NanoSEM. For the negative staining procedure, a $2 \%$ aqueous solution of ammonium molybdate tetrahydrate was prepared. Then, $20 \mu \mathrm{L}$ of sample and $20 \mu \mathrm{L}$ of staining solution were mixed and a drop of this mixture was placed onto a Formvar grid, held by tweezers. After 20 seconds, almost all the solution was removed with filter paper and left dry.

\section{Magnetic properties of magnetoliposomes}

Magnetic measurements were performed at room temperature in a Superconducting Quantum Interference Device (SQUID) magnetometer (Quantum Design MPMS5XL), using applied magnetic fields up to $5.5 \mathrm{~T}$.

The magnetization hysteresis loop measurements were performed by fixing the temperature and measuring the magnetization at a series of different applied magnetic fields. This type of study gives information about the saturation magnetization, the degree at which the sample remains magnetized when the applied field is removed, and how easily the sample magnetization can be reversed, the so-called coercive field. The magnetization was corrected for the diamagnetic contribution from the lipids and water and normalized by the mass of the samples, which was determined after drying them.

\section{Growth inhibitory activity of drug-loaded AMLs on human tumor cell lines and on porcine liver primary cells}

Two types of aqueous magnetoliposomes with different compositions (100\% Egg-PC and 95 : 5 Egg-PC : DSPE-PEG2000-Folate, from Avanti Polar Lipids) were loaded with compound 1 or compound 2, with different compound concentrations $(0.03 \mu \mathrm{M}$ to $7.5 \mu \mathrm{M})$. In vitro cytotoxicity evaluation was assessed for four human tumor cell lines, namely MCF-7 (breast adenocarcinoma), NCI-H460 (non-small cell lung cancer), HeLa (cervical carcinoma) and T3M4 (pancreatic cancer). The cell line PLP2 (non-tumor cells) was used to evaluate the toxicity to healthy tissues. The cell lines were obtained from Leibniz Institute DSMZ - German Collection of Microorganisms and Cell Cultures.

The cells were routinely maintained as adherent cell cultures in RPMI-1640 medium containing 10\% heat-inactivated FBS, at $37{ }^{\circ} \mathrm{C}$, in a humidified air incubator containing $5 \% \mathrm{CO}_{2}$. Each cell line was plated at an appropriate density $\left(1.0 \times 10^{4}\right.$ cells per well) in 96-well plates and allowed to attach for $24 \mathrm{~h}$. The cells were then treated for $48 \mathrm{~h}$ with the different solutions. Following this incubation period, the adherent cells were fixed by adding cold $10 \%$ TCA $(100 \mu \mathrm{L})$ and incubated for $60 \mathrm{~min}$ at $4{ }^{\circ} \mathrm{C}$. Plates were then washed with deionized water and dried. A sulforhodamine B (SRB) solution $(0.1 \%$ in $1 \%$ acetic acid, 100 $\mu \mathrm{L}$ ) was then added to each plate-well and incubated for $30 \mathrm{~min}$ at room temperature. Unbound SRB was removed by washing with $1 \%$ acetic acid. The plates were air-dried and the bound SRB was solubilized with $10 \mathrm{mM}$ Tris- $\mathrm{HCl}$ buffer $(200 \mu \mathrm{L}, \mathrm{pH}=$ 7.4). The absorbance was then measured at $540 \mathrm{~nm}$ in a microplate reader. ${ }^{39,40}$ The results were expressed in $\mathrm{GI}_{50}$ values (concentration that inhibited $50 \%$ of net cell growth).

\section{Results and discussion}

\section{Photophysical properties in homogeneous solution}

The promising antitumor properties of the di(hetero)arylamines 1 and 2 (Fig. 1) ${ }^{29}$ inspired us to study the photophysical 
behaviour of these compounds in homogeneous solution, as they exhibit intrinsic fluorescence. Thus, the absorption and fluorescence properties of the thieno[3,2- $b]$ pyridine derivatives $\mathbf{1}$ and $\mathbf{2}$ were studied in several solvents of different polarity. The maximum absorption $\left(\lambda_{\mathrm{abs}}\right)$ and emission wavelengths $\left(\lambda_{\mathrm{em}}\right)$, molar absorption coefficients and fluorescence quantum yields are shown in Table 1. The normalized fluorescence spectra are displayed in Fig. 3 and 4 (examples of absorption spectra are also shown).

The thieno[3,2- $b]$ pyridines 1 and 2 present moderate to high absorption coefficient values $\left(\varepsilon \geq 7 \times 10^{3} \mathrm{M}^{-1} \mathrm{~cm}^{-1}\right)$ in all solvents, having also very reasonable fluorescence quantum yields (Table 1), a similar behaviour to that observed in other thienopyridine derivatives previously studied..$^{26,34,42,43}$

Additional common features with other thienopyridines are the negligible fluorescence in protic media (due to hydrogen bonding with this type of solvents), the red shifts in emission and band enlargement with increasing solvent polarity, ${ }^{26,34,42,43}$ usually attributed to an intramolecular charge transfer (ICT) character of the excited state. ${ }^{37}$ The effect is more pronounced for compound 2, exhibiting larger bathochromic shifts in polar solvents (a red shift of $120 \mathrm{~nm}$ between cyclohexane and dimethylsulfoxide, Table 1).

The significant sensitivity of the emission of diarylamines 1 and 2 to their environment can be useful to understand their behaviour in magnetoliposomes of different compositions.

\section{Magnetoliposomes formulations}

Both aqueous (AMLs) and solid (SMLs) magnetoliposomes containing manganese ferrite nanoparticles were prepared. The influence of lipid membrane fluidity in AMLs was investigated using egg-phosphatidylcholine (Egg-PC), a fluid phosphatidylcholine mixture at room temperature ${ }^{\mathbf{4 4}}$ dipalmitoylphosphatidylcholine (DPPC), with a melting transition temperature of $41{ }^{\circ} \mathrm{C},{ }^{45}$ and Egg-PC/cholesterol $7: 3$, the latter mixture usually being used as model of biological membranes. ${ }^{46,47}$ Neat Egg-PC and EggPC : cholesterol aqueous magnetoliposomes containing lauric acid coated $\mathrm{MnFe}_{2} \mathrm{O}_{4}$ nanoparticles were also previously prepared by Pradhan and coworkers, ${ }^{48}$ the Egg-PC : cholesterol $2: 1$ being

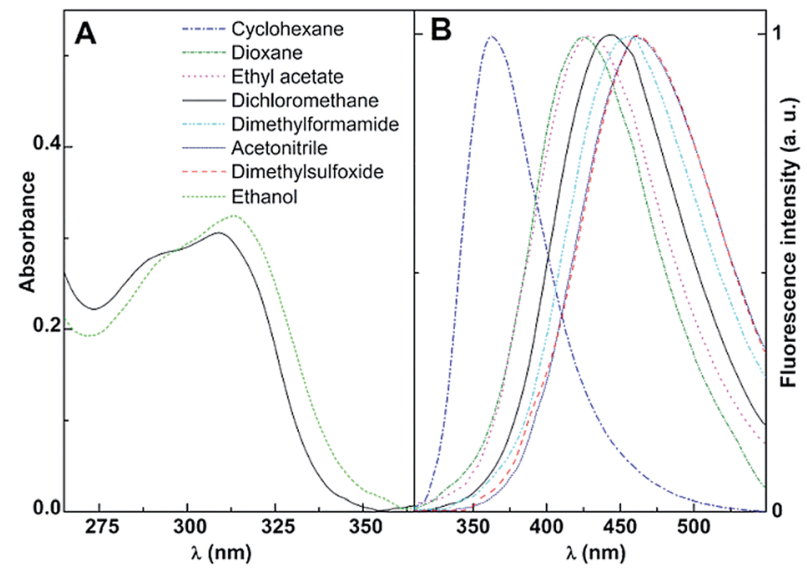

Fig. 3 (A) Absorption spectra of $2 \times 10^{-5} \mathrm{M}$ solutions of compound 1 in dichloromethane and ethanol, as examples. (B) Normalized fluorescence spectra $\left(\lambda_{\text {exc }}=310 \mathrm{~nm}\right)$ of $3 \times 10^{-6} \mathrm{M}$ solutions of compound 1 in several solvents.

the most promising formulation for hyperthermia treatment of cancer.

Here, DPPC was used for the first time in the preparation of solid magnetoliposomes containing manganese ferrite NPs. The interest of this phospholipid is its melting transition temperature slightly above the physiological temperature, thus being promising for the release of drugs enhanced by the phase transition in combined chemotherapy/hyperthermia strategies. In fact, several studies have demonstrated that the thermal enhancement of the cytotoxic activity of many anticancer agents is maximized at mild hyperthermia temperatures $\left(40.5-43{ }^{\circ} \mathrm{C}\right){ }^{49}$

The formation of the double DPPC bilayer around a cluster of magnetic nanoparticles was investigated by FRET assays. Thus, the NDB labeled lipid NBD-C ${ }_{6}$-HPC was included in the second lipid layer of SMLs (NBD acting as the energy donor), while the labeled lipid rhodamine B-DOPE (energy acceptor) was incorporated in the first lipid layer. The emission of SMLs containing both donor and acceptor labeled lipids was measured exciting only NBD, and was compared with the emission of SMLs labeled with only the energy donor (Fig. 5). Comparing the fluorescence spectra of the two systems, a decrease in the NBD emission

Table 1 Maximum absorption ( $\left.\lambda_{\text {abs }}\right)$ and emission $\left(\lambda_{\mathrm{em}}\right)$ wavelengths, molar absorption coefficients $(\varepsilon)$ and fluorescence quantum yields $\left(\Phi_{\mathrm{F}}\right)$ for the thieno[3,2-b]pyridine derivatives 1 and 2 in several solvents

\begin{tabular}{|c|c|c|c|c|c|c|}
\hline \multirow[b]{2}{*}{ Solvent } & \multicolumn{2}{|c|}{$\lambda_{\mathrm{abs}}(\mathrm{nm})\left(\varepsilon / 10^{4} \mathrm{M}^{-1} \mathrm{~cm}^{-1}\right)$} & \multicolumn{2}{|c|}{$\lambda_{\mathrm{em}}(\mathrm{nm})$} & \multicolumn{2}{|l|}{$\Phi_{\mathrm{F}}^{a}$} \\
\hline & 1 & 2 & 1 & 2 & 1 & 2 \\
\hline Dioxane & $300(1.67)$ & $282 s h ; 312(1.17)$ & 428 & 436 & 0.35 & 0.30 \\
\hline Ethyl acetate $^{b}$ & $293 \mathrm{sh} ; 302(1.01)$ & 284 (1.30); 315 (1.18) & 431 & 450 & 0.60 & 0.59 \\
\hline Dichloromethane & $293 s h ; 310(1.54)$ & $283 s h ; 312(1.15)$ & 444 & 461 & 0.53 & 0.39 \\
\hline Dimethylsulfoxide $^{b}$ & $311(1.96)$ & $313(1.39)$ & 461 & 486 & 0.48 & 0.36 \\
\hline Ethanol & $313(1.77)$ & $287 s h ; 313$ (1.06) & - & - & - & - \\
\hline
\end{tabular}

${ }^{a}$ Relative to anthracene in ethanol $\left(\Phi_{\mathrm{r}}=0.27\right) .{ }^{41}$ Error about $10 \% .{ }^{b}$ Solvents cut-off: ethyl acetate: $265 \mathrm{~nm}$; dimethylsulfoxide: 270 nm; $N, N$ dimethylformamide: $275 \mathrm{~nm}$. sh: shoulder. 


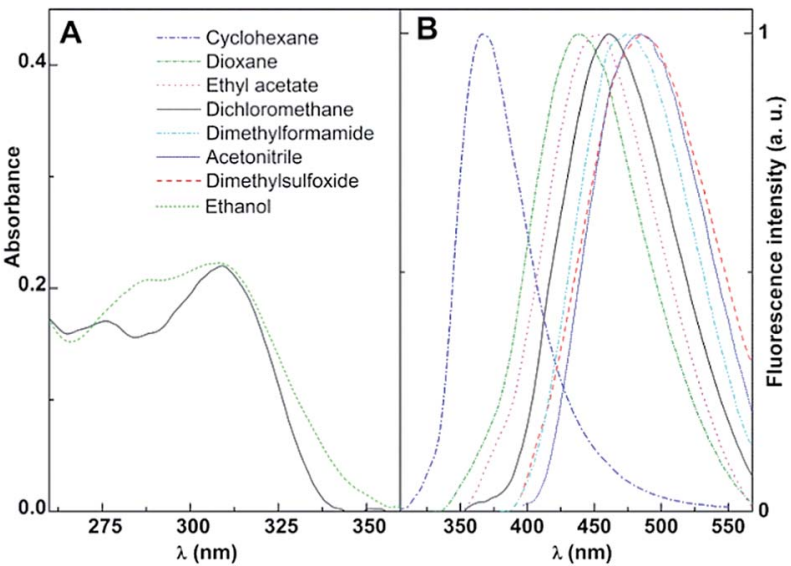

Fig. 4 (A) Absorption spectra of $2 \times 10^{-5} \mathrm{M}$ solutions of compound 2 in dichloromethane and ethanol, as examples. (B) Normalized fluorescence spectra $\left(\lambda_{\text {exc }}=310 \mathrm{~nm}\right)$ of $3 \times 10^{-6} \mathrm{M}$ solutions of compound 2 in several solvents.

band and the presence of a pronounced rhodamine emission is observed in SMLs containing both fluorophores, evidencing the energy transfer from the excited NBD to rhodamine B.

A FRET efficiency of $85 \%$ was calculated from eqn (3) to (6), with a corresponding donor-acceptor distance $(r)$ of $5.3 \mathrm{~nm}$. Considering that a cell membrane presents a thickness of 7 to $9 \mathrm{~nm},{ }^{50}$ it can be concluded that the labeled lipids rhodamineDOPE and NBD-C 6 -HPC are located in the first and second lipid layer, respectively, around the nanoparticle clusters, confirming the synthesis of the solid magnetoliposomes.

Analogous results were obtained for SMLs of DPPC : cholesterol $2: 1$. These results are also similar to the ones already reported for SMLs of the phospholipid DOPG containing manganese ferrite nanoparticles, ${ }^{31}$ indicating that the method previously developed by us for the preparation of DOPG solid magnetoliposomes ${ }^{33}$ can also be employed for different lipids. Size distributions of $124 \pm$ $23 \mathrm{~nm}$ (by number) and of $141 \pm 38 \mathrm{~nm}$ (by intensity) were obtained through Dynamic Light Scattering measurements for SMLs

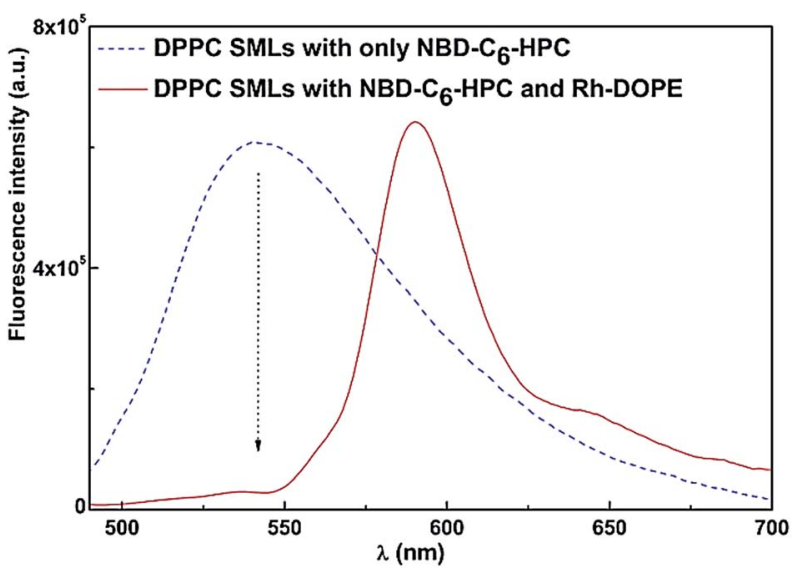

Fig. 5 Fluorescence spectra ( $\lambda_{\text {exc }}=470 \mathrm{~nm}$, no rhodamine excitation) of SMLs with DPPC bilayer labeled with only NBD- $\mathrm{C}_{6}-\mathrm{HPC}$ and SMLS labeled with both NBD- $\mathrm{C}_{6}-\mathrm{HPC}$ and rhodamine B-DOPE. of phospholipid DPPC (Fig. S1 in ESI $\dagger$ ), showing an appropriate size for biomedical applications. SEM images of these SMLs also evidence structures with diameters lower than $150 \mathrm{~nm}$ (Fig. S2 in ESI $†$ ), in accordance with DLS data.

\section{Magnetic properties of magnetoliposomes}

Previously synthesized manganese ferrite nanoparticles have shown to be superparamagnetic at room temperature. ${ }^{31}$ Here, the magnetic hysteresis cycles of aqueous magnetoliposomes and solid magnetoliposomes were measured at $316 \mathrm{~K}$ and are displayed in Fig. 6. The hysteresis loop of neat $\mathrm{MnFe}_{2} \mathrm{O}_{4} \mathrm{NPs}$ is also shown, for comparison.

In terms of hysteresis, both nanosystems present a similar behaviour to the one of neat NPs, with small coercive fields of 4.22 Oe and 8.43 Oe for SMLs and AMLs, respectively (Fig. 6). Table 2 summarizes the magnetic data obtained from the loops. The presence of superparamagnetic behaviour can be evidenced by determining the magnetic squareness value, which is the ratio between the remnant magnetization, $M_{\mathrm{r}}$, and the saturation magnetization, $M_{\mathrm{s}}$. Magnetic squareness values indicative of superparamagnetism are of the order or below 0.1, meaning the loss of more than $90 \%$ of the magnetization upon removal of the applied magnetic field. ${ }^{51,52}$ The magnetic squareness values for AMLs and SMLs clearly indicate the presence of a superparamagnetic behaviour (Table 2).

As previously reported for magnetoliposomes based on iron oxide nanoparticles, ${ }^{53,54}$ the saturation magnetization of the manganese ferrite nanoparticles decreases after encapsulation into liposomes, this decrease being much more pronounced in the case of AMLs. For SMLs, the saturation magnetization is close to the one of neat $\mathrm{MnFe}_{2} \mathrm{O}_{4}$ nanoparticles, while for AMLs a strong decrease is observed (Table 2). This is due to the lower molar ratio between magnetic NPs and lipids for AMLs, when compared to SMLs and bare NPs samples, which contributes to an overall lower saturation magnetization of the combined lipid + nanoparticles system. On the other hand, on AMLs and SMLs,

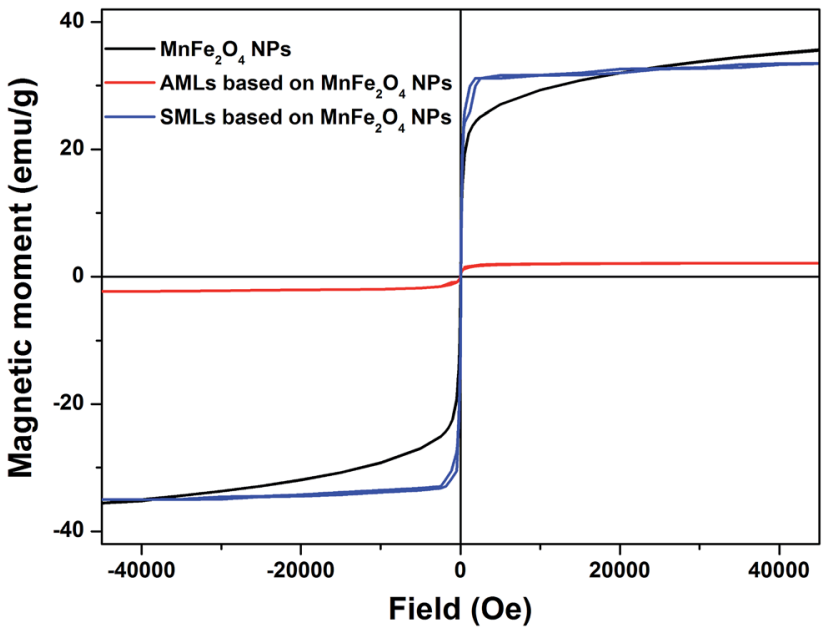

Fig. 6 Magnetization hysteresis cycles, measured at $316 \mathrm{~K}$, of neat $\mathrm{MnFe}_{2} \mathrm{O}_{4}$ nanoparticles, DPPC SMLs and Egg-PC AMLs containing $\mathrm{MnFe}_{2} \mathrm{O}_{4} \mathrm{NPs}$. 
Table 2 Coercive field $\left(H_{\mathrm{c}}\right)$, saturation magnetization $\left(M_{\mathrm{s}}\right)$, remnant magnetization $\left(M_{r}\right)$ and ratio $M_{r} / M_{s}$ for manganese ferrite NPs and magnetoliposomes

\begin{tabular}{lllll}
\hline System & $H_{\mathrm{c}}(\mathrm{Oe})$ & $M_{\mathrm{s}}\left(\mathrm{emu} \mathrm{g}^{-1}\right)$ & $M_{\mathrm{r}}\left(\mathrm{emu} \mathrm{g}^{-1}\right)$ & $M_{\mathrm{r}} / M_{\mathrm{s}}$ \\
\hline $\mathrm{MnFe}_{2} \mathrm{O}_{4} \mathrm{NPs}^{29}$ & 6.30 & 36.00 & 0.58 & 0.016 \\
$\mathrm{SMLs} \mathrm{(DPPC)}_{\text {AMLs (Egg-PC) }}$ & 4.22 & 34.16 & 0.90 & 0.03 \\
& & 1.17 & 0.08 & 0.07 \\
\hline
\end{tabular}

a more clear saturation of the hysteresis loops is observed as compared to the bare NPs (Fig. 6). This indicates an increased degree of clustering of the nanoparticles encapsulated in liposomes, which enhances the interparticle magnetic interaction and favors the alignment of their magnetic moments, as is more clearly observed at higher applied magnetic fields.

\section{Fluorescence studies in (magneto)liposomes}

The thienopyridine derivatives $\mathbf{1}$ and $\mathbf{2}$ were encapsulated in magnetoliposomes, both aqueous (AMLs) and solid (SMLs). For comparison, studies in liposomes (without magnetic nanoparticles) of the same lipid compositions were also performed.

Examples of fluorescence emission spectra of compounds 1 and 2 in magnetoliposomes are shown in Fig. 7 (maximum emission wavelengths are presented in Table 3). Emission in liposomes (without magnetic nanoparticles and with the same concentration of compound) is also shown for comparison. A quenching effect of compounds emission by the magnetic nanoparticles is observed, proving the incorporation of these potential drugs in the magnetic nanocarriers. As expected, the fluorescence quenching is significantly more pronounced in solid magnetoliposomes, where the magnetic nanoparticles are closer to the antitumor compounds. This behaviour is similar to the previously observed with another antitumor thienopyridine derivative in AMLs and SMLs containing $\mathrm{MnFe}_{2} \mathrm{O}_{4} \mathrm{NPs}^{31}$

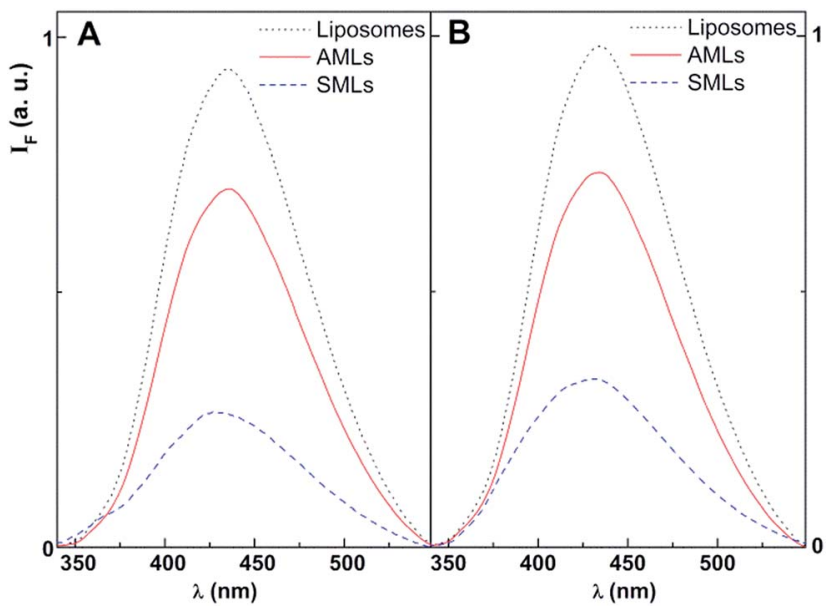

Fig. 7 Normalized fluorescence spectra of compounds 1 and 2 (3 $\times$ $10^{-6} \mathrm{M}$ ) in liposomes and magnetoliposomes (Egg-PC AMLs and DPPC SMLs), at room temperature $\left(\lambda_{\text {exc }}=310 \mathrm{~nm}\right)$. (A) Compound 1; (B) compound 2 .
Relevant information about the location of these antitumor compounds in (magneto)liposomes can be obtained through fluorescence anisotropy ( $r$ ) measurements, as the $r$ value increases with the rotational correlational time of the fluorescent molecule (and, thus, with the viscosity of the environment, eqn $(8))^{37}$

$$
\frac{1}{r}=\frac{1}{r_{0}}\left(1+\frac{\tau}{\tau_{\mathrm{c}}}\right)
$$

where $r_{0}$ is the fundamental anisotropy, $\tau$ is the excited-state lifetime and $\tau_{\mathrm{c}}$ is the rotational correlation time.

The fluorescence (steady-state) anisotropies of these diarylamines in the several systems are displayed in Table 3. For comparison, the fluorescence anisotropy values in the highly viscous solvent glycerol $\left(\eta=993.4 \mathrm{cP} \text { at } 25{ }^{\circ} \mathrm{C}\right)^{55}$ at room temperature were also measured (Table 3). Upon temperature increase, the excited-state lifetime of these molecules is predicted to decrease, due to the increment of the non-radiative deactivation pathways (mainly the rate constant for internal conversion $S_{1} \rightarrow S_{0}$ ). According to eqn (8), an increase of the fluorescence anisotropy is expected from a diminution of the excited-state lifetime. The anisotropy values for $\mathbf{1}$ and $\mathbf{2}$ in DPPC-based systems decrease significantly at $55{ }^{\circ} \mathrm{C}$ (Table 3 ) and this behaviour can be attributed to a decrease in the rotational correlation time of the fluorophore. This originates from the decrease of membrane microviscosity upon changing from the gel to the liquid-crystalline phase of DPPC above $41{ }^{\circ} \mathrm{C}$ (melting transition temperature).

The differences in anisotropy values between the compounds incorporated in lipid systems and in glycerol at room temperature can also be attributed to the distinct environment viscosities, as lipid membranes, exhibiting viscosity values around $100-200 \mathrm{cP}, 56,57$ are much less viscous than glycerol. These results indicate that both compounds are mainly located in the lipid bilayers.

The effect of cholesterol (Ch) in Egg-PC membranes, as reported by fluorescence anisotropy values, is different for compounds 1 and 2 (a slight increase in fluidity reported by compound 2 and a diminution for compound 1). However, this effect is small and may be related with slightly different locations of these compounds in membranes. From the values of maximum emission wavelengths in AMLs and SMLs, an environment with polarity similar to dioxane or ethyl acetate is anticipated (Tables 1 and 3).

It was reported that membrane viscosity decreases from the outside to inside the membrane ${ }^{58,59}$ and this may justify, besides possible differences in compound excited-state lifetimes, the lower anisotropies for both compounds in solid magnetoliposomes, when compared to AMLs. Maximum emission wavelength values point to less polar environments felt by compounds in SMLs (Table 3), corroborating that these potential drugs are deeply located in SMLs membranes.

The encapsulation efficiencies of both compounds in AMLs and SMLs are presented in Table 4 . Values of encapsulation efficiency are larger than $75 \%$, the lowest EE\% being observed for compound $\mathbf{2}$ in aqueous magnetoliposomes. 
Table 3 Steady-state fluorescence anisotropy $(r)$ values and maximum emission wavelengths $\left(\lambda_{\text {em }}\right)$ for diarylamines 1 and 2 in several liposome and magnetoliposome formulations, at $25^{\circ} \mathrm{C}$ and $55^{\circ} \mathrm{C}$

\begin{tabular}{|c|c|c|c|c|c|c|}
\hline & \multirow[b]{2}{*}{ Lipid formulation } & \multirow[b]{2}{*}{$T\left({ }^{\circ} \mathrm{C}\right)$} & \multicolumn{2}{|c|}{$\lambda_{\mathrm{em}}(\mathrm{nm})$} & \multicolumn{2}{|l|}{$r$} \\
\hline & & & 1 & 2 & 1 & 2 \\
\hline & Egg-PC : Ch $(7: 3)$ & 25 & 433 & 434 & 0.212 & 0.202 \\
\hline & DPPC $(100 \%)$ & 25 & 432 & 433 & 0.200 & 0.222 \\
\hline & & 55 & 430 & 431 & 0.141 & 0.101 \\
\hline & DPPC $(100 \%)$ & 25 & 432 & 433 & 0.202 & 0.287 \\
\hline & & 55 & 432 & 432 & 0.153 & 0.140 \\
\hline \multirow[t]{2}{*}{ SMLs } & DPPC (100\%) & 25 & 430 & 427 & 0.159 & 0.135 \\
\hline & & 55 & 428 & 424 & 0.101 & 0.051 \\
\hline Glycerol & - & 25 & - & - & 0.304 & 0.321 \\
\hline
\end{tabular}

These high encapsulation efficiencies point to a promising use of these nanocarriers in magnetically-guided transport of compounds $\mathbf{1}$ and 2, and as agents for simultaneous chemotherapy and hyperthermia in oncological therapy. These potentialities will allow reducing the blood circulating time of the drugs and lowering side effects, which is especially important as both compounds are hydrophobic and present some cytotoxicity for normal PLP2 cells, with low $\mathrm{GI}_{50}$ values of $1.94 \pm 0.16 \mu \mathrm{M}$ for compound 1 and $6.56 \pm 0.2 \mu \mathrm{M}$ for compound $2 .^{29}$

\section{Growth inhibitory activity of drug-loaded AMLs on human tumor cell lines and on porcine liver primary cells}

The tumor cell growth inhibitory activity of AMLs containing compounds 1 and 2 was evaluated in four human tumor cell lines, specifically MCF-7 (breast adenocarcinoma), HeLa (cervical carcinoma), T3M4 (pancreatic cancer) and NCIH460 (non-small cell lung cancer). Moreover, the toxicity of the drug-loaded AMLs towards non-tumor cells was studied using porcine liver primary cells (PLP2). This allowed the determination of the $\mathrm{GI}_{50}$ values $(\mu \mathrm{M})$, corresponding to the compound concentration which inhibited $50 \%$ of cell growth (Table 5). Besides the neat Egg-PC AMLs, the influence of 5\% PEG-folate in AMLs formulation was also investigated, as folate receptors are generally overexpressed in tumor cells.

Both aqueous and solid magnetoliposomes (without compounds) evidenced no inhibitory activity on the same cell

Table 4 Encapsulation efficiencies (EE\%) of compounds 1 and 2 in magnetoliposomes (SD: standard deviation)

\begin{tabular}{lll}
\hline & EE\% \pm SD & \\
\cline { 2 - 3 } System & Compound 1 & Compound 2 \\
\hline AMLs (Egg-PC) & $89.0 \pm 4.5$ & $75.9 \pm 6.8$ \\
SMLs (DPPC) & $88.1 \pm 4.9$ & $82.1 \pm 6.1$
\end{tabular}

lines, including the non-tumor PLP2, when tested in the same conditions used with the drug-loaded systems. From the results obtained (Table 5), it can be concluded that the drugloaded AMLs are efficient in inhibiting tumor cells, presenting $\mathrm{GI}_{50}$ values similar to the ones determined previously using only the compounds, ${ }^{29}$ except for compound 2 in these formulations, that presents very much low $\mathrm{GI}_{50}$ values for the NCI-H460 lung cancer cell line. The presence of folate has only a significant effect for compound $\mathbf{1}$ in HeLa tumor cell line. For compound 2, the presence of folate in magnetoliposomes does not influence noticeably the results (Table 5). Nevertheless, the $\mathrm{GI}_{50}$ values clearly show that aqueous magnetoliposomes are able to release the encapsulated compounds $\mathbf{1}$ and $\mathbf{2}$ and to inhibit the growth of tumor cells. It is also noteworthy the very low $\mathrm{GI}_{50}$ values obtained with compounds $\mathbf{1}$ and $\mathbf{2}$ using the pancreatic tumor cell line T3M4, where the single compounds had not been previously tested.

In general, the results in PLP2 cells (Table 5) show that the encapsulated compounds are not toxic for the $\mathrm{GI}_{50}$ values in all tumor cell lines, apart from the compound 2-loaded systems in MCF-7 cells, that present $\mathrm{GI}_{50}$ values higher than for PLP2.

The low $\mathrm{GI}_{50}$ values obtained for non-tumor PLP2 cells reinforce the importance of using a magnetic field gradient to guide and target these nanosystems for tumors in vivo, taking also advantage of hyperthermia capability.

Unfortunately, for the drug-loaded DPPC solid magnetoliposomes (SMLs), no satisfactory results were obtained, with very high $\mathrm{GI}_{50}$ values (above $7.5 \mu \mathrm{M}$ ) in all tumor cell lines (Table S1 in ESI $\dagger$ ). This is possibly due to the fact that the phospholipid DPPC is in the rigid gel phase at room temperature, thus preventing the release of the compounds. The addition of 5\% DSPE-PEG-Folate did not improve the results (Table $\mathrm{S} 1 \dagger$ ). Other lipid formulations for SMLs will be tested in the near future, as well as assays at mild hyperthermia temperatures will be carried out. Also, confocal microscopy measurements will be performed to assess internalization of magnetoliposomes in cells. 
Table 5 Growth inhibitory activity of drug-loaded aqueous magnetoliposomes on various human tumor cell lines and non-tumor porcine liver primary cells (PLP2)

\begin{tabular}{|c|c|c|c|c|c|c|}
\hline \multirow[b]{2}{*}{ Cell line } & \multicolumn{3}{|c|}{$\mathrm{GI}_{50}$ values $^{a}(\mu \mathrm{M})$ for compound $\mathbf{1}$} & \multicolumn{3}{|c|}{$\mathrm{GI}_{50}$ values $^{a}(\mu \mathrm{M})$ for compound 2} \\
\hline & $\begin{array}{l}\text { Only compound } 1 \\
\text { (ref. 29) }\end{array}$ & $\begin{array}{l}\text { Egg-PC AMLs with } \\
\text { compound } 1\end{array}$ & $\begin{array}{l}\text { Egg-PC/PEG-Fol (95:5) } \\
\text { AMLs with compound } 1\end{array}$ & $\begin{array}{l}\text { Only compound } 2 \\
\text { (ref. 29) }\end{array}$ & $\begin{array}{l}\text { Egg-PC AMLs with } \\
\text { compound } 2\end{array}$ & $\begin{array}{l}\text { Egg-PC/PEG-Fol (95: 5) } \\
\text { AMLs with compound } 2\end{array}$ \\
\hline HeLa & $0.09 \pm 0.00$ & $0.15 \pm 0.02$ & $0.09 \pm 0.01$ & $1.40 \pm 0.06$ & $1.32 \pm 0.15$ & $1.44 \pm 0.07$ \\
\hline MCF7 & $0.25 \pm 0.03$ & $0.45 \pm 0.01$ & $0.44 \pm 0.0003$ & $5.88 \pm 0.86$ & $5.67 \pm 0.62$ & $5.56 \pm 0.11$ \\
\hline T3M4 & - & $0.26 \pm 0.02$ & $0.30 \pm 0.002$ & - & $1.56 \pm 0.05$ & $1.68 \pm 0.07$ \\
\hline
\end{tabular}

${ }^{a} \mathrm{GI}_{50}$ values correspond to the concentration which inhibited $50 \%$ of cell growth. Results are from three independent experiments (performed in triplicate), and are expressed as mean \pm standard deviation (SD).

\section{Conclusions}

In this work, the fluorescence emission of two new promising antitumor compounds, a $N$-(3-methoxyphenyl)thieno[3,2-b]pyridin7-amine 1 and a $N$-(2-methoxyphenyl)thieno[3,2-b]pyridin-7amine 2, was studied in different environments. These in vitro potent antitumor thienopyridin-7-arylamine derivatives were successfully incorporated in both aqueous and solid magnetoliposomes based on superparamagnetic manganese ferrite nanoparticles, with high encapsulation efficiencies. The intrinsic fluorescence of the potential antitumor drugs was used as a tool to obtain information about compounds location in magnetoliposomes.

The magnetic properties of the manganese ferrite-based magnetoliposomes were measured for the first time, evidencing a superparamagnetic behaviour.

Growth inhibitory concentrations on tumor cell lines obtained with aqueous magnetoliposomes with encapsulated compounds 1 and 2 point to promising future drug delivery applications of these anticancer compounds in vivo, using magnetoliposomes simultaneously as drug nanocarriers and hyperthermia agents, to increase the efficacy of anticancer therapies.

\section{Acknowledgements}

This work was supported by the Portuguese Foundation for Science and Technology (FCT) in the framework of the Strategic Funding of CF-UM-UP (UID/FIS/04650/2013), CQUM (UID/QUI/00686/2013 and 2016) and CIMO (UID/AGR/00690/ 2013), the latter also supported by FEDER - Program PT2020. FCT, POPH-QREN and FSE are acknowledged for the PhD grant of A. R. O. Rodrigues (SFRH/BD/90949/2012), the postdoc grant of R. C. Calhelha (SFRH/BPD/68344/2010) and for financial support to MAP-Fis PhD Programme.

\section{Notes and references}

1 S. Mornet, S. Vasseur, F. Grasset and E. Duguet, Magnetic nanoparticle design for medical diagnosis and therapy, $J$. Mater. Chem., 2004, 14, 2161-2175.
2 S. Dandamudi and R. B. Campbell, Development and characterization of magnetic cationic liposomes for targeting tumor microvasculature, Biochim. Biophys. Acta, 2007, 1768, 427-438.

3 A. S. Lübbe, C. Bergemann, J. Brock and D. G. McClure, Physiological aspects in magnetic drug targeting, J. Magn. Magn. Mater., 1999, 194, 149-155.

4 S. Dandamudi and R. B. Campbell, The drug loading, cytotoxicity and tumor vascular targeting characteristics of magnetite in magnetic drug targeting, Biomaterials, 2007, 28, 4673-4683.

5 H.-J. Weinmann, W. Ebert, B. Misselwitz and H. S. -Willich, Tissue-specific MR contrast agents, Eur. J. Radiol., 2003, 46, 33-44.

6 N. Nuytten, M. Hakimhashemi, T. Ysenbaert, L. Defour, J. Trekker, S. J. Soenen, P. Van der Meeren and M. Cuyper, PEGylated lipids impede the lateral diffusion of adsorbed proteins at the surface of (magneto)liposomes, Colloids Surf., B, 2010, 80, 227-231.

7 U. I. Tromsdorf, N. C. Bigall, M. G. Kaul, O. T. Bruns, M. S. Nikolic, B. Mollwitz, R. A. Sperling, R. Reimer, H. Hohenberg, W. J. Parak, S. Forster, U. Beisiegel, G. Adam and H. Weller, Size and surface effects on the MRI relaxivity of manganese ferrite nanoparticle contrast agents, Nano Lett., 2007, 7, 2422-2427.

8 E. Amstad, J. Kohlbrecher, E. Müller, T. Schweizer, M. Textor and E. Reimhult, Triggered release from liposomes through magnetic actuation of iron oxide nanoparticle containing membranes, Nano Lett., 2011, 11, 1664-1670.

9 P. Tartaj, M. P. Morales, S. Veintemillas-Verdaguer, T. Gonzalez-Carreno and C. J. Serna, The preparation of magnetic nanoparticles for applications in biomedicine, J. Phys. D: Appl. Phys., 2003, 36, 182-197.

10 M. Faraji, Y. Yamini and M. Rezaee, Magnetic nanoparticles: synthesis, stabilization, functionalization, characterization, and applications, J. Iran. Chem. Soc., 2010, 7, 1-37.

11 C. M. Niemeyer, Nanoparticles, proteins, and nucleic acids: biotechnology meets materials science, Angew. Chem., Int. Ed., 2001, 40, 4128-4158.

12 G. Gregoriadis, Engineering liposomes for drug delivery, Trends Biotechnol., 1995, 13, 527-537. 
13 I. M. Hafez, S. Ansell and P. R. Cullis, Tunable pH-sensitive liposomes composed of mixtures of cationic and anionic lipids, Biophys. J., 2000, 79, 1438-1446.

14 J. B. Sun, J. H. Duan, S. L. Dai, J. Ren, L. Guo, W. Jiang and Y. Li, Preparation and anti-tumor efficiency evaluation of doxorubicin-loaded bacterial magnetosomes: magnetic nanoparticles as drug carriers isolated from magnetospirillum gryphiswaldense, Biotechnol. Bioeng., 2008, 101, 1313-1320.

15 Y. Malam, M. Loizidou and A. M. Seifalian, Liposomes and nanoparticles: nanosized vehicles for drug delivery in cancer, Trends Pharmacol. Sci., 2009, 30, 592-599.

16 M. J. Munchhof, J. S. Beebe, J. M. Casavant, B. A. Cooper, J. L. Doty, R. C. Higdon, S. M. Hillerman, C. I. Soderstrom, E. A. Knauth, M. A. Marx, A. M. K. Rossi, S. B. Sobolov and J. Sun, Design and SAR of thienopyrimidine and thienopyridine inhibitors of VEGFR-2 kinase activity, Bioorg. Med. Chem. Lett., 2004, 14, 21-24.

17 H. R. Heyman, R. R. Frey, P. F. Bousquet, G. A. Cunha, M. D. Moskey, A. A. Ahmed, N. B. Soni, P. A. Marcotte, L. J. Pease, K. B. Glaser, M. Yates, J. J. Bouska, D. H. Albert, C. L. Black-Schaefer, P. J. Dandliker, K. D. Stewart, P. Rafferty, S. K. Davidsen, M. R. Michaelides and M. L. Curtin, Thienopyridine urea inhibitors of KDR kinase, Bioorg. Med. Chem. Lett., 2007, 17, 1246-1249.

18 S. Claridge, F. Raeppel, M.-C. Granger, N. Bernstein, O. Saavedra, L. Zhan, D. Llewellyn, A. Wahhab, R. Deziel, J. Rahil, N. Beaulieu, H. Nguyen, I. Dupont, A. Barsalou, C. Beaulieu, I. Chute, S. Gravel, M.-F. Robert, S. Lefebvre, M. Dubay, R. Pascal, J. Gillespie, Z. Jin, J. Wang, J. M. Besterman, A. R. MacLeod and A. Vaisburg, Discovery of a novel and potent series of thieno[3,2- $b]$ pyridine-based inhibitors of $c$-Met and VEGFR-2 tyrosine kinases, Bioorg. Med. Chem. Lett., 2008, 18, 2793-2798.

19 O. Saavedra, S. Claridge, L. Zhan, F. Raeppel, M.-C. Granger, S. Raeppel, M. Mannion, F. Gaudette, N. Zhou, L. Isakovic, N. Bernstein, R. Déziel, H. Nguyen, N. Beaulieu, C. Beaulieu, I. Dupont, J. Wang, R. Macleod, J. M. Besterman and A. Vaisburg, $\mathrm{N}^{3}$-Arylmalonamides: a new series of thieno[3,2- $b]$ pyridine based inhibitors of $c$ Met and VEGFR2 tyrosine kinases, Bioorg. Med. Chem. Lett., 2009, 19, 6836-6839.

20 S. Raeppel, S. Claridge, O. Saavedra, F. Gaudette, L. Zhan, M. Mannion, N. Zhou, F. Raeppel, M.-C. Granger, L. Isakovic, R. Déziel, H. Nguyen, N. Beaulieu, C. Beaulieu, I. Dupont, M.-F. Robert, S. Lefebvre, M. Dubay, J. Rahil, J. Wang, H. Ste-Croix, A. R. Macleod, J. Besterman and A. Vaisburg, $\quad N$-(3-Fluoro-4-(2-arylthieno[3,2- $b]$ pyridin-7yloxy)phenyl)-2-oxo-3-phenyl-imidazolidine-1-carboxamides: a novel series of dual $c$-Met/VEGFR2 receptor tyrosine kinase inhibitors, Bioorg. Med. Chem. Lett., 2009, 19, 1323-1328.

21 V. A. Machado, D. Peixoto, R. Costa, H. J. C. Froufe, R. C. Calhelha, R. M. V. Abreu, I. C. F. R. Ferreira, R. Soares and M.-J. R. P. Queiroz, Synthesis, antiangiogenesis evaluation and molecular docking studies of 1-aryl-3[(thieno[3,2-b]pyridin-7-ylthio)phenyl]ureas: discovery of a new substitution pattern for type II VEGFR-2 Tyr kinase inhibitors, Bioorg. Med. Chem., 2015, 23, 6497-6509.
22 I. Hayakawa, R. Shioya, T. Agatsuma, H. Furukawa and Y. Sugano, Thienopyridine and benzofuran derivatives as potent anti-tumor agents possessing different structureactivity relationships, Bioorg. Med. Chem. Lett., 2004, 14, 3411-3414.

23 M.-J. R. P. Queiroz, R. C. Calhelha, L. A. Vale-Silva, E. Pinto and M. S.-J. Nascimento, Novel 6-[(hetero)arylamino]thieno $[3,2-b]$ pyridines: synthesis and antitumoral activities, Eur. J. Med. Chem., 2010, 45, 5732-5738.

24 M.-J. R. P. Queiroz, R. C. Calhelha, L. A. Vale-Silva, E. Pinto, R. T. Lima and M. H. Vasconcelos, Efficient synthesis of new 6-(hetero)arylthieno[3,2- $b]$ pyridines by Suzuki-Miyaura coupling. Antitumoral evaluation and SARs, Eur. J. Med. Chem., 2010, 45, 5628-5634.

25 M.-J. R. P. Queiroz, R. C. Calhelha, L. A. Vale-Silva, E. Pinto, G. M. Almeida and M. H. Vasconcelos, Synthesis and evaluation of tumor cell growth inhibition of novel methyl 3-amino-6-[(hetero)arylethynyl] thieno[3,2-b]pyridine-2-

carboxylates. Structure-activity relationships and effects on the cell cycle and apoptosis, Eur. J. Med. Chem., 2011, 46, 236-240.

26 M.-J. R. P. Queiroz, S. Dias, D. Peixoto, A. R. O. Rodrigues, A. D. S. Oliveira, P. J. G. Coutinho, L. A. Vale-Silva, E. Pinto and E. M. S. Castanheira, New potential antitumoral di(hetero)-arylether derivatives in the thieno[3,2- $b]$ pyridine series: synthesis and fluorescence studies in solution and in nanoliposomes, J. Photochem. Photobiol., A, 2012, 238, 71-80.

27 R. M. V. Abreu, I. C. F. R. Ferreira, R. C. Calhelha, R. T. Lima, M. H. Vasconcelos, F. Adega, R. Chaves and M.-J. R. P. Queiroz, Anti-hepatocellular carcinoma activity using HepG2 cells and hepatotoxicity of 6-substituted methyl 3aminothieno[3,2-b]pyridine-2-carboxylate derivatives: in vitro evaluation, cell cycle analysis and QSAR studies, Eur. J. Med. Chem., 2011, 46, 5800-5806.

28 R. C. Calhelha, I. C. F. R. Ferreira, D. Peixoto, R. M. V. Abreu, L. A. Vale-Silva, E. Pinto, R. T. Lima, M. I. Alvelos, M. H. Vasconcelos and M.-J. R. P. Queiroz, Aminodi(hetero)aryl-amines in the thieno[3,2- $b]$ pyridine series: synthesis, effects in human tumor cells growth, cell cycle analysis, apoptosis and evaluation of toxicity using non-tumor cells, Molecules, 2012, 17, 3834-3843.

29 M.-J. R. P. Queiroz, D. Peixoto, R. C. Calhelha, P. Soares, T. Santos, R. T. Lima, J. F. Campos, R. M. V. Abreu, I. C. F. R. Ferreira and M. H. Vasconcelos, New di(hetero) arylethers and di(hetero)arylamines in the thieno[3,2- $b]$ pyridine series: synthesis, growth inhibitory activity on human tumor cell lines and non-tumor cells, effects on cell cycle and on programmed cell death, Eur. J. Med. Chem., 2013, 69, 855-862.

30 V. A. Machado, D. Peixoto, M.-J. R. P. Queiroz and R. Soares, Antiangiogenic 1-aryl-3-[3-(thieno[3,2-b]pyridin-7-ylthio)phenyl]ureas inhibit MCF-7 and MDA-MB-231 human breast cancer cell lines through PI3K/Akt and MAPK/Erk pathways, J. Cell. Biochem., 2016, 117, 2791-2799.

31 A. R. O. Rodrigues, J. M. F. Ramos, I. T. Gomes, B. G. Almeida, J. P. Araújo, M.-J. R. P. Queiroz, 
P. J. G. Coutinho and E. M. S. Castanheira, Magnetoliposomes based on manganese ferrite nanoparticles as nanocarriers for antitumor drugs, $R S C$ Adv., 2016, 6, 17302-17313.

32 J. M. H. Kremer, M. W. J. V. D. Esker, C. Pathmamanoharan and P. H. Wiersema, Vesicles of variable diameter prepared by a modified injection method, Biochemistry, 1977, 16, 3932-3935.

33 A. R. O. Rodrigues, I. T. Gomes, B. G. Almeida, J. P. Araújo, E. M. S. Castanheira and P. J. G. Coutinho, Magnetoliposomes based on nickel ferrite nanoparticles for biomedical applications, Phys. Chem. Chem. Phys., 2015, 17, 18011-18021.

34 C. N. C. Costa, A. C. L. Hortelão, J. M. F. Ramos, A. D. S. Oliveira, R. C. Calhelha, M.-J. R. P. Queiroz, P. J. G. Coutinho and E. M. S. Castanheira, A new antitumoral heteroarylaminothieno[3,2-b]pyridine derivative: its incorporation into liposomes and interaction with proteins monitored by fluorescence, Photochem. Photobiol. Sci., 2014, 13, 1730-1740.

35 J. N. Demas and G. A. Crosby, The measurement of photoluminescence quantum yields. - Review, J. Phys. Chem., 1971, 75, 991-1024.

36 S. Fery-Forgues and D. Lavabre, Are fluorescence quantum yields so tricky to measure? A demonstration using familiar stationery products, J. Chem. Educ., 1999, 76, 1260-1264.

37 B. Valeur, Molecular Fluorescence - Principles and Applications, Wiley-VCH, Weinheim, 2002.

38 I. Johnson and M. T. Z. Spence, Molecular Probes Handbook: A Guide to Fluorescent Probes and Labeling Technologies, Invitrogen, 11th edn, 2011.

39 M. I. Dias, L. Barros, M. Dueñas, E. Pereira, A. M. Carvalho, R. C. Alves, M. B. P. P. Oliveira, C. Santos-Buelga and I. C. F. R. Ferreira, Chemical composition of wild and commercial Achillea millefolium L. and bioactivity of the methanolic extract, infusion and decoction, Food Chem., 2013, 141, 4152-4160.

$40 \mathrm{~V}$. Vichai and K. Kirtikara, Sulforhodamine B colorimetric assay for cytotoxicity screening, Nat. Protoc., 2006, 1, 1112-1116.

$41 \mathrm{~W}$. Dawson and M. Windsor, Fluorescence yields of aromatic compounds, J. Phys. Chem., 1968, 72, 3251-3260.

42 M.-J. R. P. Queiroz, D. Peixoto, A. R. O. Rodrigues, P. Mendes, C. N. C. Costa, P. J. G. Coutinho and E. M. S. Castanheira, New 1,3-diarylureas linked by C-C Suzuki coupling to the methyl 3-aminothieno[3,2- $b]$ pyridine-2-carboxylate moiety: synthesis and fluorescence studies in solution and in lipid membranes, J. Photochem. Photobiol., A, 2013, 255, 27-35.

43 M. S. D. Carvalho, A. C. L. Hortelão, R. C. Calhelha, A. S. Abreu, P. J. G. Coutinho, M.-J. R. P. Queiroz and E. M. S. Castanheira, Fluorescence studies on potential antitumor 6-(hetero) arylthieno[3,2-b]pyridine derivatives in solution and in nanoliposomes, J. Photochem. Photobiol., A, 2013, 264, 56-66.

44 D. Papahadjopoulos and N. Miller, Phospholipid model membranes. I. Structural characteristics of hydrated liquid crystals, Biochim. Biophys. Acta, 1967, 135, 624-638.
45 B. Lentz, Membrane 'fluidity' as detected by diphenylhexatriene probes, Chem. Phys. Lipids, 1989, 50, 171-190.

46 C. Toniolo, M. Crisma, F. Formaggio, C. Peggion, V. Monaco, C. Goulard, S. Rebuffat and B. Bodo, Effect of $N^{\alpha}$-acyl chain length on the membrane-modifying properties of synthetic analogs of the lipopeptaibol trichogin GA IV, J. Am. Chem. Soc., 1996, 118, 4952-4958.

47 M. Lucaciu, S. Rebuffat, C. Goulard, H. Duclohier, G. Molle and B. Bodo, Interaction of the 14-residue peptaibols, harzianins HC, with lipid bilayers: permeability modifications and conductance properties, Biochim. Biophys. Acta, 1997, 1323, 85-96.

48 P. Pradhan, J. Giri, R. Banerjee, J. Bellare and D. Bahadur, Preparation and characterization of manganese ferritebased magnetic liposomes for hyperthermia treatment of cancer, J. Magn. Magn. Mater., 2007, 311, 208-215.

49 A. Hervault and N. T. K. Thanh, Magnetic nanoparticlebased therapeutic agents for thermo-chemotherapy treatment of cancer, Nanoscale, 2014, 6, 11553-11573.

$50 \mathrm{H}$. Curtis and N. Barnes, Biology, Worth Publishers, New York, 5th edn, 1989.

51 D. L. Schulz, R. A. Sailer and A. N. Caruso, Superparamagnetic transition metal iron oxygen nanoparticles, US Pat., No. 0194733, 2009.

52 L. Khanna and N. K. Verma, Synthesis, characterization and in vitro cytotoxicity study of calcium ferrite nanoparticles, Mater. Sci. Semicond. Process., 2013, 16, 1842-1848.

53 S. Zhang, H. Niu, Y. Zhang, J. Liu, Y. Shia, X. Zhang and Y. Cai, Biocompatible phosphatidylcholine bilayer coated on magnetic nanoparticles and their application in the extraction of several polycyclic aromatic hydrocarbons from environmental water and milk samples, $J$. Chromatogr. A, 2012, 1238, 38-45.

54 S. García-Jimeno, E. Escribano, J. Queralt and J. Estelrich, Magnetoliposomes prepared by reverse-phase followed by sequential extrusion: characterization and possibilities in the treatment of inflammation, Int. J. Pharm., 2011, 405, 181-187.

55 CRC Handbook of Chemistry and Physics, ed. D. R. Lide, CRC Press/Taylor and Francis, Boca Raton, FL, USA, 89th edn, 2009.

56 J. N. Israelachvili, S. Marcelja and R. G. Horn, Physical principles of membrane organization, Q. Rev. Biophys., 1980, 13, 121-200.

57 D. B. Kell and C. M. Harris, On the dielectrically observable consequences of the diffusional motions of lipids and proteins in membranes. 1. Theory and overview, Eur. Biophys. J., 1985, 12, 181-197.

58 L. Tilley, K. R. Thulborn and W. H. Sawyer, An assessment of the fluidity gradient of the lipid bilayer as determined by a set of $n$-(9-anthroyloxy)fatty acids ( $n=2,6,9,12,16), J$. Biol. Chem., 1979, 254, 2592-2594.

59 M. A. Bahri, B. J. Heyne, P. Hans, A. E. Seret, A. A. MouithysMickalad and M. D. Hoebeke, Quantification of lipid bilayer effective microviscosity and fluidity effect induced by propofol, Biophys. Chem., 2005, 114, 53-61. 